\title{
ON INTERVALS OF PRESCRIBED LENGTHS
}

\section{ANTHONY P. MORSE}

The present paper is an outgrowth of a conversation with Maurice Sion. In this conversation he asked whether the closed unit interval always contains a set of Lebesgue measure zero which cannot be embraced by a sequence of open intervals of prescribed lengths adding up to 1 . I posed the corresponding question for the unit open interval. Answers to these questions are given by Theorems 3 and 6 below.

Let us agree: $\omega$ is the set of non-negative integers; a sequence is a function whose domain is $\omega ; S$ embraces $A$ if and only if $S$ is such a sequence of sets that

$$
A \subset \cup_{n \in \omega} S_{n}
$$

$\mathcal{L}$ is (outer) Lebesgue measure; $J$ is a $\lambda$ file if and only if $J$ is such a sequence of open intervals and $\lambda$ is such a sequence of non-negative real numbers that

$$
\begin{aligned}
\mathcal{L}\left(J_{n}\right) & =\lambda_{n} \\
I & =\mathrm{E} t(0<t<1) ; \quad \text { whenever } n \in \omega ; \\
I^{\prime} & =\mathrm{E} t(0 \leqq t \leqq 1) ;
\end{aligned}
$$

$S$ is separate if and only if $S$ is such a sequence of sets that

$$
S_{m} \cap S_{n}=0 \quad \text { whenever } m \in \omega, n \in \omega, m \neq n ;
$$

subsum $\lambda$ is the set of numbers of the form

$$
\sum_{n \in \alpha} \lambda_{n}
$$

where $\alpha \subset \omega$.

Lemma 1 below is of considerable use to us here and is easily seen with the aid of E. M. Beesley and A. P. Morse, $\phi$-Cantorian functions and their convex moduli, Duke Math. J. vol. 12 (1945) p. 608, Theorem 7.1.

1. Lemma. If $\lambda$ is a sequence of non-negative real numbers,

$$
\sum_{n} \in_{\omega} \lambda_{n}<1 \text {, }
$$

then $I$ contains such a set $A$ of Lebesgue measure zero that no $\lambda$ file embraces $A$.

2. THEOREM, If $\lambda$ is a sequence of non-negative real numbers,

Received by the editors November 16, 1953. 


$$
\sum_{n \in \omega} \lambda_{n}=1,
$$

then I contains such a set $A$ of Lebesgue measure zero that

$$
J \text { is separate and } \bigcup_{n} \in{ }_{\omega} J_{n} \subset I
$$

whenever $J$ is a $\lambda$ file which embraces $A$.

Proof. Let $F$ be the family of such sequences $\mu$ that for some $k \in \omega$ :

$$
\mu_{n}=\lambda_{n} \quad \text { whenever } n \in \omega \text { and } n \neq k \text {; }
$$

$\mu_{k}$ is rational and $0<\mu_{k}<\lambda_{k}$.

Clearly $F$ is countable. Furthermore each $\mu \in F$ is such a sequence of non-negative real numbers that

$$
\sum_{n \in \omega} \mu_{n}<1 .
$$

Consequently Lemma 1 and the principle of choice enable us to associate with each $\mu \in F$ such a set $B_{\mu}$ that:

$$
B_{\mu} \subset I, \quad \mathcal{L}\left(B_{\mu}\right)=0,
$$

$$
\text { no } \mu \text { file embraces } B_{\mu} \text {. }
$$

Now let

$$
A=\bigcup_{\mu} \in_{F} B_{\mu} .
$$

Clearly

$$
A \subset I, \quad \mathcal{L}(A)=0 ;
$$

and completion of the proof is tantamount to verification of the

Statement. If $J$ is any $\lambda$ file which embraces $A$ then

$$
J \text { is separate and } \bigcup_{n \in \omega} J_{n} \subset I \text {. }
$$

Proof. Suppose the contrary and so choose $p \in \omega$ that either:

$$
0 \neq J_{p} \cap J_{n} \quad \text { and } \sup J_{p} \leqq \sup J_{n}
$$

for some $n \in \omega$ with $n \neq p$; or

$$
J_{p} \nsubseteq I .
$$

In either event it is clearly possible to so ascertain a sequence $K$ of open intervals that:

$$
\begin{array}{lr}
K_{n}=J_{n} & \text { whenever } n \in \omega \text { with } n \neq p ; \\
K_{p} \subset J_{p} ; & \text { is rational and } 0<\mathcal{L}\left(K_{p}\right)<\mathcal{L}\left(J_{p}\right) ; \\
\mathcal{L}\left(K_{p}\right) \quad
\end{array}
$$




$$
\begin{aligned}
& I \cap \bigcup_{n} \in{ }_{\omega} K_{n}=I \cap \bigcup_{n} \in_{\omega} J_{n} ; \\
& K \operatorname{embraces} A .
\end{aligned}
$$

Letting $\nu$ be the sequence for which

$$
\nu_{n}=\mathcal{L}\left(K_{n}\right) \quad \text { whenever } n \in \omega
$$

we see at once that

$$
\nu \in F, \quad K \text { is a } \nu \text { file which embraces } A .
$$

Hence, in contradiction to (1),

$$
\nu \in F, K \text { is a } \nu \text { file which embraces } B_{\nu} .
$$

3. TheOREm. If $\lambda$ is a sequence of non-negative real numbers,

$$
\sum_{n \in \omega} \lambda_{n}=1 \text {, }
$$

then $I^{\prime}$ contains such a set $A^{\prime}$ of Lebesgue measure zero that no $\lambda$ file embraces $A^{\prime}$.

Proof. Choose $A$ in accordance with Theorem 2 and form $A^{\prime}$ by adjoining 0 to $A$.

4. ThEOREM. If $\lambda$ is a sequence of non-negative real numbers,

$$
\begin{aligned}
& 0<\lambda_{0}<1, \\
& \sum_{n \in \omega} \lambda_{n}=1, \\
& H=\operatorname{subsum} \lambda, \\
& \mathcal{L}(H)=0,
\end{aligned}
$$

then $I$ contains such a set $B$ of Lebesgue measure zero that no $\lambda$ file embraces $B$.

Proof. Choose $A$ in accordance with Theorem 2 and let

$$
B=A \cup(H \cap I) \text {. }
$$

Clearly

$$
\mathcal{L}(B)=0 \text { and } B \subset I \text {; }
$$

that no $\lambda$ file embraces $B$ is a consequence of the

Statement. If $J$ is a $\lambda$ file which embraces $A$ then $J$ does not embrace $H \cap I$.

Proof. Since $J$ embraces $A$ we infer from Theorem 2 that

$$
J \text { is separate and } U_{n} \in \omega_{\omega} J_{n} \subset I \text {. }
$$

Because of this and the fact that 


$$
0<\lambda_{0}<1
$$

we choose such an end point $\xi$ of $J_{0}$ that

$$
\xi \in I \text {. }
$$

Since $J$ is separate we are sure

$$
\xi \in \bigcup_{n} \in \omega_{\omega} J_{n} .
$$

Now let

$$
\begin{array}{ll}
a=\mathrm{E} t(t<\xi), & b=\mathrm{E} t(\xi<t), \\
\alpha=\mathrm{E} n \in \omega\left[J_{n} \subset a\right], & \beta=\mathrm{E} n \in \omega\left[J_{n} \subset b\right] .
\end{array}
$$

Because of (2) it is evident that

$$
\alpha \cup \beta=\omega
$$

and, because of (1), it follows that

$$
\begin{aligned}
0 & \geqq \sum_{n \in \alpha} \mathcal{L}\left(J_{n}\right)-\xi \\
& \geqq \sum_{n \in \alpha} \mathcal{L}\left(J_{n}\right)-\xi+\sum_{n \in \beta} \mathcal{L}\left(J_{n}\right)-1+\xi \\
& \geqq \sum_{n} \in \omega \\
& =\sum_{n}\left(J_{n}\right)-1 \\
& =0 .
\end{aligned}
$$

Consequently

$$
\xi=\sum_{n \in \alpha} \mathcal{L}\left(J_{n}\right)=\sum_{n \in \alpha} \lambda_{n}
$$

and thus

$$
\xi \in H \cap I .
$$

Reference to (2) now convinces us that $J$ does not embrace $H \cap I$.

5. THEOREM. If $\lambda$ is a sequence of non-negative real numbers,

$$
\begin{aligned}
& \sum_{n \in \omega} \lambda_{n}=1, \\
& H=\operatorname{subsum} \lambda, \\
& \mathcal{L}(H)>0, \\
& A \subset I, \\
& \mathcal{L}(A)=0,
\end{aligned}
$$

then there is a $\lambda$ file which embraces $A$.

Proof. Let us agree herein that 


$$
T^{*}(W)
$$

is the image under the function $T$ of the set $W$. Let us also agree herein that $R$ is the set of real finite numbers.

Let $C$ be the set of such functions $T$ on $R$ that for some $n \in \omega$

$$
T(x)=x+\lambda_{n} \quad \text { whenever } x \in R .
$$

Let $G$ be the smallest transformation group which contains $C$. Since $C$ is a countable set of translations of $R$ into itself it follows that $G$ is also a countable set of translations of $R$ into itself.

We let

$$
B=\cup_{T \in G} T^{*}(A)
$$

and divide the remainder of the proof into seven parts. Of these Part I is obvious, Part II is an immediate consequence of the countability of $G$, Parts III and IV are, no doubt, special cases of well known general theorems, and Part VII is the conclusion desired.

PART I. $A \subset I \cap B$.

PART II. $\mathcal{L}(B)=0$.

PART III. If $S \in G$ then $S^{*}(B) \subset B$.

Proof.

$$
\begin{aligned}
S^{*}(B) & =S^{*}\left(\bigcup_{T \in G} T^{*}(A)\right) \\
& =\cup_{T} \in_{G} S^{*}\left(T^{*}(A)\right) \\
& \subset B .
\end{aligned}
$$

PART IV. If $T \in G$ then $T^{*}(R \sim B)=R \sim B$.

Proof. Let $P$ be the inverse of $T$. From Part III we learn

$$
P^{*}(B) \subset B \text {. }
$$

Hence

$$
B=T^{*}\left(P^{*}(B)\right) \subset T^{*}(B)
$$

and re-use of Part III assures us

$$
T^{*}(B)=B \text {. }
$$

Accordingly

$$
T^{*}(R \sim B)=T^{*}(R) \sim T^{*}(B)=R \sim B .
$$

PART V. If $n \in \omega, x \in R \sim B, r^{2}=1$, then

$$
x+r \cdot \lambda_{n} \in R \sim B .
$$

Proof. Use Part IV. 
Easily deduced from Part V is

PART VI. If $\gamma$ is a finite subset of $\omega, x \in R \sim B, r^{2}=1$, then

$$
x+r \cdot \sum_{m \in \gamma} \lambda_{m} \in R \sim B .
$$

Part VII. Some $\lambda$ file embraces $A$.

Proof. Since $\mathcal{L}(B)=0$ we are sure

$$
\mathcal{L}(H \sim B)=\mathcal{L}(H)>0,
$$

and we so choose $\xi$ that

$$
\xi \in H \sim B,
$$

and then so choose $\alpha \subset \omega$ that

$$
\xi=\sum_{n} \in{ }_{\alpha} \lambda_{n}
$$

Let

$$
\beta=\omega \sim \alpha
$$

and note

$$
1-\xi=\sum_{n \in \beta} \lambda_{n}
$$

For each $n \in \omega$ let:

$$
\begin{gathered}
M_{n}=\mathrm{E} m \in \alpha[0 \leqq m<n] ; \\
N_{n}=\mathrm{E} m \in \beta[0 \leqq m<n] .
\end{gathered}
$$

Let $J$ be such a sequence that:

$$
J_{n}=\mathrm{E} t\left[\xi-\lambda_{n}<t+\sum_{m} \in M_{n} \lambda_{m}<\xi\right]
$$

whenever $n \in \alpha$;

$$
J_{n}=\mathrm{E} t\left[\xi<t-\sum_{m \in N_{n}} \lambda_{m}<\xi+\lambda_{n}\right]
$$

whenever $n \in \beta$.

Clearly $J$ is a $\lambda$ file.

If $0<x \leqq \xi$ and

$$
p=\sup \mathrm{E}_{n} \in_{\omega}\left[x+\sum_{m \in M_{n}} \lambda_{m} \leqq \xi\right]
$$

then $p \in \alpha$ and either

$$
x \in J_{p} \quad \text { or } \quad x=\xi-\sum_{m \in M_{p}} \lambda_{m} .
$$

Similarly if $\xi \leqq x<1$ and

$$
q=\sup \mathrm{E}_{n} \in \omega\left[\xi \leqq x-\sum_{m} \in N_{n} \lambda_{m}\right]
$$

then $q \in \beta$ and either 


$$
x \in J_{q} \text { or } x=\xi+\sum_{m \in N_{q}} \lambda_{m} .
$$

From the last two paragraphs, from Part VI, and from the fact that

$$
\xi \in H \sim B \subset R \sim B
$$

it follows that

$$
I \subset R \sim B \cup \cup_{n} \in{ }_{\omega} J_{n} .
$$

According to this and Part I

$$
A \subset I \cap B \subset \cup_{n} \in \omega_{\omega} J_{n}
$$

and

$$
J \text { embraces } A \text {. }
$$

Since it has already been noted that $J$ is a $\lambda$ file the proof of Part VII is now complete.

6. THEOREM. If $\lambda$ is a sequence of non-negative real numbers,

$$
\begin{aligned}
& 0<\lambda_{0}<1, \\
& \sum_{n \in \omega} \lambda_{n}=1, \\
& H=\text { subsum } \lambda,
\end{aligned}
$$

then a necessary and sufficient condition that I contains such a set $A$ of Lebesgue measure zero that no $\lambda$ file embraces $A$ is that

$$
\mathcal{L}(H)=0 .
$$

Proof. Use Theorems 4 and 5.

7. Remarks. If $\lambda$ is such a sequence that

$$
\lambda_{n}=2^{-1} \cdot 2^{-n}
$$

whenever $n \in \omega$

then

$$
\sum_{n} \in{ }_{\omega} \lambda_{n}=1
$$

and subsum $\lambda=I^{\prime}$.

Less obvious is the fact that if $\lambda$ is such a sequence that

$$
\lambda_{n}=3 \cdot 10^{-1} \cdot\left(2^{-n}+4^{-n}\right) \quad \text { whenever } n \in \omega
$$

then

$$
\sum_{n} \in{ }_{\omega} \lambda_{n}=1
$$

and subsum $\lambda$ is a nondense perfect set of Lebesgue measure $3 / 5$. 
Finally if $\lambda$ is such a sequence that

$$
\lambda_{n}=2 \cdot 3^{-1} \cdot 3^{-n} \quad \text { whenever } n \in \omega
$$

then subsum $\lambda$ is the Cantor ternary set of Lebesgue measure zero.

University of California, Berkeley

\section{BOUNDS FOR THE MODULI OF THE ZEROS OF A POLYNOMIAL}

FULTON KOEHLER

1. Introduction. Let $f(z)=a_{0}+a_{1} z+\cdots+a_{n} z^{n}, a_{0} \neq 0, a_{n} \neq 0$. Following the notation of Ostrowski [1], we define the Newton diagram of $f(z)$ as the broken line $D$ in the $(x, y)$-plane with the following properties:

(1) $D$ extends from the point $x=0, y=-\log \left|a_{0}\right|$ to the point $x=n, y=-\log \left|a_{n}\right|$;

(2) $y_{\nu} \leqq\left(y_{\nu-1}+y_{\nu+1}\right) / 2(\nu=1,2, \cdots, n-1)$, where $y_{\nu}$ is the ordinate of $D$ for $x=\nu$;

(3) The points $\left(\nu,-\log \left|a_{\nu}\right|\right)(\nu=0,1, \cdots, n)$ all lie on or above $D$;

(4) If $\left(\nu, y_{\nu}\right)$ is a corner point of $D$, i.e., $y_{\nu}<\left(y_{\nu-1}+y_{\nu+1}\right) / 2$, then $y_{\nu}=-\log \left|a_{\nu}\right|$.

Values of $\nu$ for which $\left(\nu,-\log \left|a_{\nu}\right|\right)$ is a corner point or an end point of $D$ are called principal indices of $f(z)$. Let $y_{\nu}=-\log T_{\nu}$ so $T_{\nu}=\left|a_{\nu}\right|$ when $\nu$ is a principal index, and let $R_{\nu}=T_{\nu-1} / T_{\nu}$. The slope of $D$ in the interval $(\nu-1, \nu)$ is $\log R_{\nu}$.

Let the zeros of $f(z)$ be denoted by $z_{1}, z_{2}, \cdots, z_{n}$ with $0<\left|z_{1}\right|$ $\leqq\left|z_{2}\right| \leqq \cdots \leqq\left|z_{n}\right|$, and let $b_{\boldsymbol{k}}^{n}$ and $B_{\boldsymbol{k}}^{n}(k=1,2, \cdots, n)$ be the minimum and maximum respectively of $\left|z_{k}\right| / R_{k}$ for all polynomials of degree $n$ which do not vanish at the origin. Ostrowski [1] has shown, by considering the polynomial $z^{n} f(1 / z)$, that

$$
B_{k}^{n}=1 / b_{n-k+1}^{n} \text {. }
$$

Ostrowski proved that $b_{1}^{n}$ is the positive root of the equation $1=x$ $+x^{2}+\cdots+x^{n}$, that $b_{n}^{n}$ is equal to $1 / n$, and that

Presented to the Society, April 24, 1953; received by the editors March 10, 1953 and, in revised form, September 28, 1953. 